\title{
Performance and Durability of Adhesive Tapes for Building Applications. From Product Documentation to Scientific Knowledge (and Back Again)
}

\author{
Malin Sletnes ${ }^{1}$ and Susanne Frank ${ }^{2}$ \\ ${ }^{1}$ SINTEF Community, Høgskoleringen 7B, 7034, Trondheim, Norway, malin.sletnes@sintef.no \\ 2 SINTEF Community, Høgskoleringen 7B, 7034, Trondheim, Norway, susanne.frank@sintef.no
}

\begin{abstract}
Adhesive tapes are used extensively in both new buildings and renovation projects in order to achieve airtight building envelopes and energy efficient buildings. It is important to be able to reliably predict whether these adhesive solutions are durable. In our roles as both a research institute and a national approval body for building materials, SINTEF have been performing product evaluations and testing of adhesive tapes for 10 years. During this time, we have tested the durability of more than 30 different tapes on a variety of substrates commonly used in the building industry. After systemising and analysing this data, we have gained valuable insight into the factors governing the durability of adhesive tapes for the construction market. In this paper we share our findings and discuss how we intend to utilise the new knowledge in the design of further scientific experiments in the field.
\end{abstract}

Keywords: Adhesive Tape, Construction, Durability, Accelerated Ageing.

\section{Introduction}

Air-tightening systems, such as vapour barriers and wind barriers are essential for energy efficient buildings. In order to achieve high air-tightness performance, the overlaps and connections of the air-tightening systems to other building components like foundations, roof constructions, windows, and air and electric ducts, are today frequently sealed by selfadhesive tapes. However, there is little knowledge on the durability of the adhesive tapes used in construction, partly because it is a relatively new application, and partly because it is a very complex system to study systematically in laboratory conditions (Leprince et al., 2017) due to the many variables in the properties of adhesives and substrates, as well as the effect of initial conditions of the bond formation and climatic exposure.

In response to this knowledge gap several research groups have recently studied the durability of air-tightness systems including adhesive products. (Antonsen, 2017; Fufa et al., 2018; Langmans et al., 2017; Litvak et al., 2019) The referenced papers all include welldefined, systematic studies, many of them focusing on full- or reduced-scale airtightness system testing before and after artificial ageing. However, the small number of adhesive tapes included in each study restricts the possibility to draw general conclusions. Often no more than two tapes are tested per system. This is a necessary limitation, due to the extensive experimental matrix that would otherwise arise if tapes should also be tested on several different substrates and under different exposure conditions.

The main objective of the research project TightEN $(2019-2023)$ is to develop robust test and evaluation methodology for ensuring durable adhesive airtight solutions for energy efficient building envelopes. The experimental design at the starting phase of a project will be 
important for the outcome, and prior knowledge of important parameters can result in a better design of the experimental matrix. Thus, we have systemised and analysed our existing data on adhesive tape durability in order to gain a better understanding of the importance of different parameters. In our roles as both a research institute and a national approval body for building materials, SINTEF have been performing product evaluations and testing of adhesive tapes for 10 years, testing more than 30 tapes. The data we have available is mostly from small-scale adhesive bond testing, such as peel and shear resistance of the joint. The correlation of these properties with the actual air-tightness performance is not well established and will be investigated more thoroughly in the TightEN project. Nevertheless, we believe that adhesive joint testing will in all probability still have a role in the evaluation of adhesive tapes for airtightness application because it is simple, cost-effective and standardised.

\section{Data and Methodology}

The data subject to analysis was collected from standardised peel and shear resistance testing of adhesive joints before and after artificial ageing, over a course of 10 years. Data from both product certification and previous research projects have been included. Some tapes have been tested several times, as a part of the control procedures for product certifications. An overview of the test methods, number of tapes and associated data points included in the study are given in Table 1 .

Material testing and sample preparation was performed according to the European test

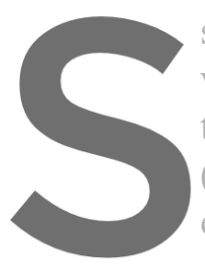
standards for peel a waterproofing, EN 123 the materials together (This is not specified earliest dates in the $180^{\circ}$ peel for tapes adthered to rigid substrates, d shear resistance of joints for plas
and applying hand force. The resultin
the test standards and may have variec
-year time period.) Peel resistance was
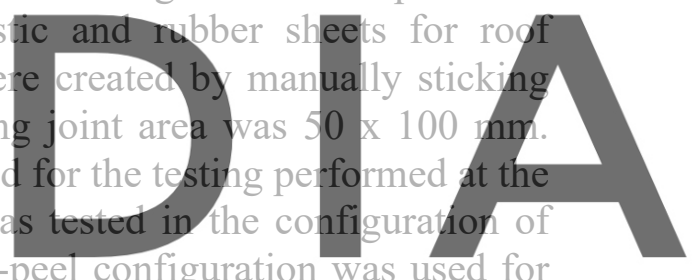
flexible substrates, such as vapour. and wind barrier membranes. The test configurations for

The test specimens were exposed to artificial ageing according to one of the three different ageing programs shown in Tabie 1 . The climate carousel refers to ageing in a vertical climate simulator according to NT Build 495. In the climate carousel, the samples are subjected in turn to four different climate exposure conditions; ultraviolet (UV) and infrared irradiation (black panel temperature of $63{ }^{\circ} \mathrm{C}$ ), water spray, freezing $\left(-20{ }^{\circ} \mathrm{C}\right)$ and ambient laboratory conditions. The exposure time is $1 \mathrm{~h}$ for each climate condition.

Table 1. Key information on the dataset subject to analysis.

\begin{tabular}{llll}
\hline Evaluation parameter & Test method & No. of unique tapes & No. of data points* \\
\hline Average peel resistance & EN 12316-2 & $\begin{array}{l}37 \\
(30 \text { acrylic, } 7 \text { butyl })\end{array}$ & 143 \\
\hline Shear resistance & EN 12317-2 & $\begin{array}{l}38 \\
(30 \text { acrylic, } 8 \text { butyl })\end{array}$ & 132 \\
\hline
\end{tabular}

* One data point includes the measurement values from both aged and non-aged samples. Each value represents the average of measurements on 5 individual samples (excluding outliers). 


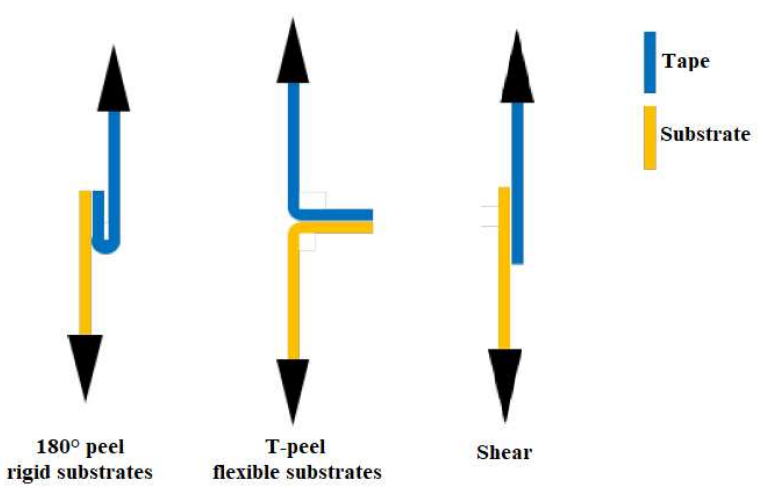

Figure 1. Configurations for testing peel and shear resistance.

Table 2. Ageing programs.

\begin{tabular}{lccc} 
Ageing program & Climate carousel & UV light & Heat chamber at $70{ }^{\circ} \mathrm{C}$ \\
\hline Climate simulator + heat & 2 or 4 weeks & - & 12,22 or 24 weeks \\
UV fluorescent lamps theat & & 2 days & 12 weeks \\
Only heat ageing & - & - & 24 weeks
\end{tabular}

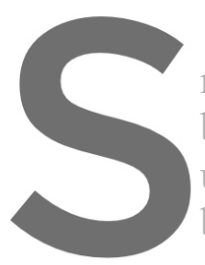

The tapes have been andnymised and dategorisec bas

material, intended application temperature ra

used, and in general the choice of substrates

based on the intended application

of a specific product range, for instance as part of an airtightness system, it would typically

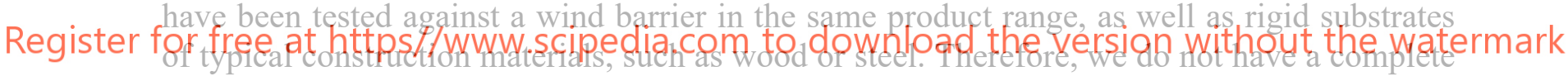
experimental matrix to analyse, and any attempts at performing rigid statistical analysis have been hampered by confounding variables and missing data points. Hence, the analysis is based on plotting and visualisation techniques.

\section{Results and Discussion}

\subsection{Repeatability of the Data}

The European test methods do not specify how the joint should be made, other than stating that the pieces should be joined by the methods to be used for installation in the end use application. I.e. application pressure and speed were not controlled. Nevertheless, the repeatability of the measurements was generally acceptable. The pooled standard deviation of measurement values within one test series ( 5 measurements on test specimens prepared from the same tape and substrates, undergoing identical ageing programs and conditioning, tested on the same day) was $2 \mathrm{~N} / 50 \mathrm{~mm}$ before ageing and $4 \mathrm{~N} / 50 \mathrm{~mm}$ after ageing for peel resistance, and $19 \mathrm{~N} / 50 \mathrm{~mm}$ before ageing and $23 \mathrm{~N} / \mathrm{mm}$ after ageing for shear resistance. 


\subsection{Correlation Between Measurements on Aged and Non-Aged Specimens}

Peel resistance for aged specimens are plotted as a function of the peel resistance for the corresponding non-aged specimens in Figure 2a. The equivalent plot for shear resistance is shown in $2 \mathrm{~b}$. The light grey fields mark the regions where the peel or shear resistance decreased with ageing, whereas the darker grey fields mark the region where the said properties decreased more than $50 \%$ relative to non-aged samples.

The purpose of the plots was to see if a correlation could be found between measurement values before and after ageing, i.e. if a high measurement value before ageing increased the probability of getting a high measurement value after aging. To some extent, this correlation is present, because large increases in joint strength after ageing were rarely observed. However, it is clear from Figure 2a, that peel resistance for non-aged samples is a poor predictor of peel resistance after ageing. In comparison, a much stronger correlation is observed for shear resistance (2b). In fact, shear resistance appears to have changed very little with ageing for a large portion of the test specimens.

The peel resistance is often seen as a measurement of the adhesion force between the adhesive and the substrate (presuming the failure mode is interfacial), whereas the shear resistance is considered to to a larger degree reflect the cohesive strength of the adhesive. Although this is a great simplification, the results can nevertheless be an indication that the degradation caused by the artificial ageing is more detrimental to the interfacial bond than to the bulk properties of the adhesive.

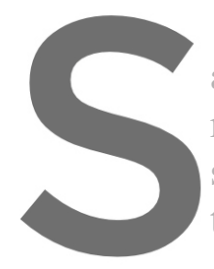

Another observation

ageing were from tape rather than rigid substrate substrate, but more like the ageing of the substrat For the design of experiments to investiga
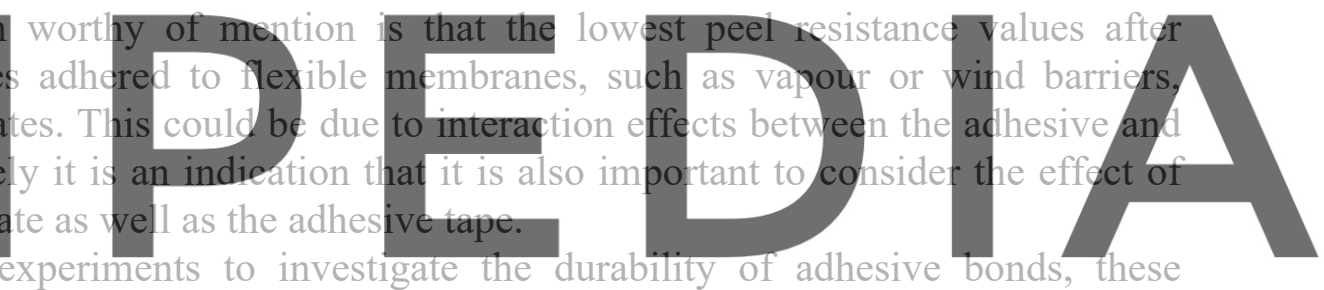

findings highlight that peel resistance is a much more sensitive evaluation parameter than

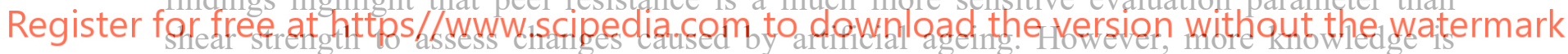

needed on both correlation between natural and artificial ageing for adhesive tapes, as well as the acting forces in in-use condition to assess whether peel or shear resistance are good indicators for long-term performance.

\subsection{Difference in Ageing Properties Across Adhesive Tape Products}

Several attempts were made to group the available test results based on substrate types and surface materials, properties of the adhesive tape products (e.g. adhesive, backing material etc.) and investigate the effect on adhesive properties, however the result was always that the range of measurement values within the groups was considerably larger than the observed difference between the groups. The difference from one unique tape to another proved to be a confounding variable for all other attempted groupings. Thus, the conclusion was drawn that the effect of ageing and substrates had to be analysed on an individual tape basis. Because this could not easily and clearly be presented for all tapes in the dataset, a selection was done according to the following criteria: 

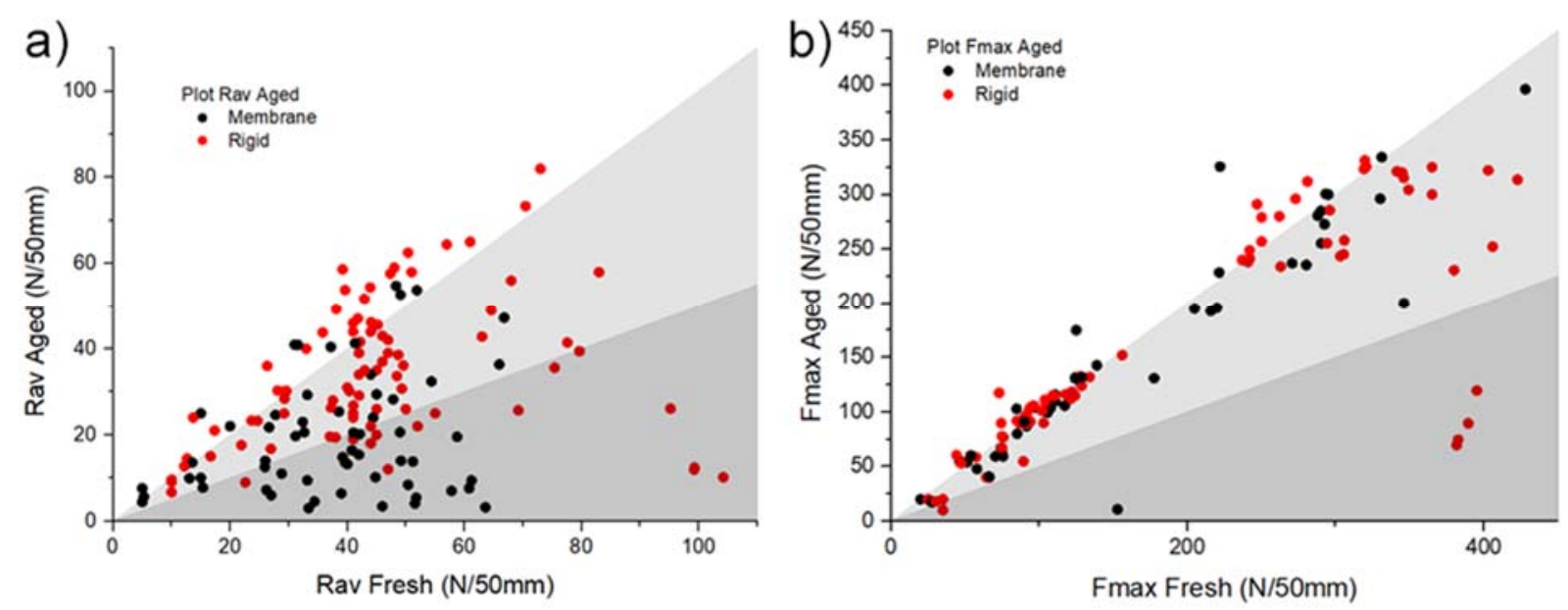

Figure 2. a) Peel resistance for aged specimens as a function of peel resistance for non-aged specimens b) Shear resistance for aged specimens as a function of shear resistance for non-aged specimens. The light and dark grey fields mark the regions where the shear resistance decreased $0-50 \%$ and $50-100 \%$, respectively

Only specimens subjected to 2 weeks climate carousel +24 weeks heat ageing

Only single-sided tapes

Only tapes tested on at least two different substrates

Excluding data points from substrates that could not be confidently categorized

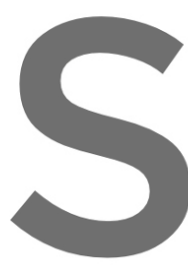

The resulting plots

respectively. In the plot

A8 for acrylic tapes and

full range of values for

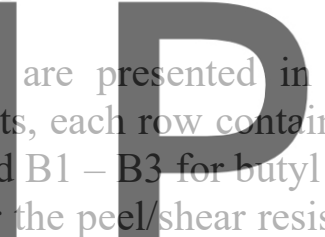

Figures 3 and 4
s data points from
ruber tapes. The 1
tance in the datase
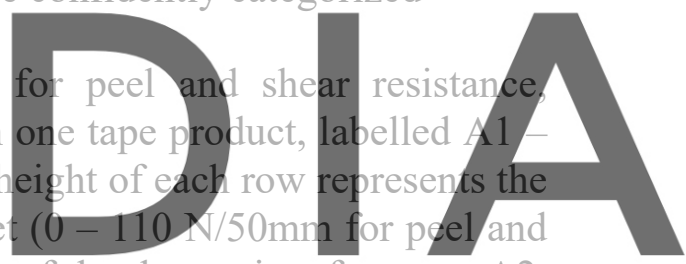

$0-450 \mathrm{~N} / 50 \mathrm{~mm}$ for shear resistance). A more detailed view of the data points for tapes A2,

Register for free at hteps/ywWW.Scipedia. Some important pbservations are:

Both initial values and change with ageing differ greatly between tapes. This can be exemplified by studying the peel resistance of tapes A2, A3 and A4. Both A2 and A3 start out at medium peel resistance for all tested substrates before ageing. After ageing, the peel resistance for A2 is largely unchanged for all substrates, wheras the values for A3 drop somewhat for all substrates, and the drop is distinctly larger for the joint to the vapour barrier than to the other substrates. A4, on the other hand starts out with peel values at the very top of the range, but degrade dramatically upon ageing for all substrates.

- The trends in initial values and degradation upon ageing are in many cases similar for peel and shear resistance. For instance A4 displays a similar drop in shear resistance upon ageing as in peel resistance.

- The effect from testing on different substrates is less pronounced. With few exceptions, all values from one tape on different substrates are in the same range. This applies to both peel and shear resistance, and both before and after ageing, however the variation from substrate to substrate appears to be slightly higher after ageing. 
All these tapes have been subjected to the same ageing program, yet they display completely different responses to the accelerated ageing. Thus, when designing experiments to learn more about the ageing mechanisms of adhesive tapes it is important to include enough different tape products to get the full picture. Furthermore, it is necessary to gain a better understanding of the differences between the tapes, and what properties are significant for the durability.

The number of substrates may be kept smaller to limit the size of the experimental matrix. However, it is important to do the ageing on stable, rigid substrates as well as membranes to account for the fact that membranes themselves are also subject to changing properties during the ageing process. If fact, membrane properties should possibly also be checked seperately, in order to correct for this.

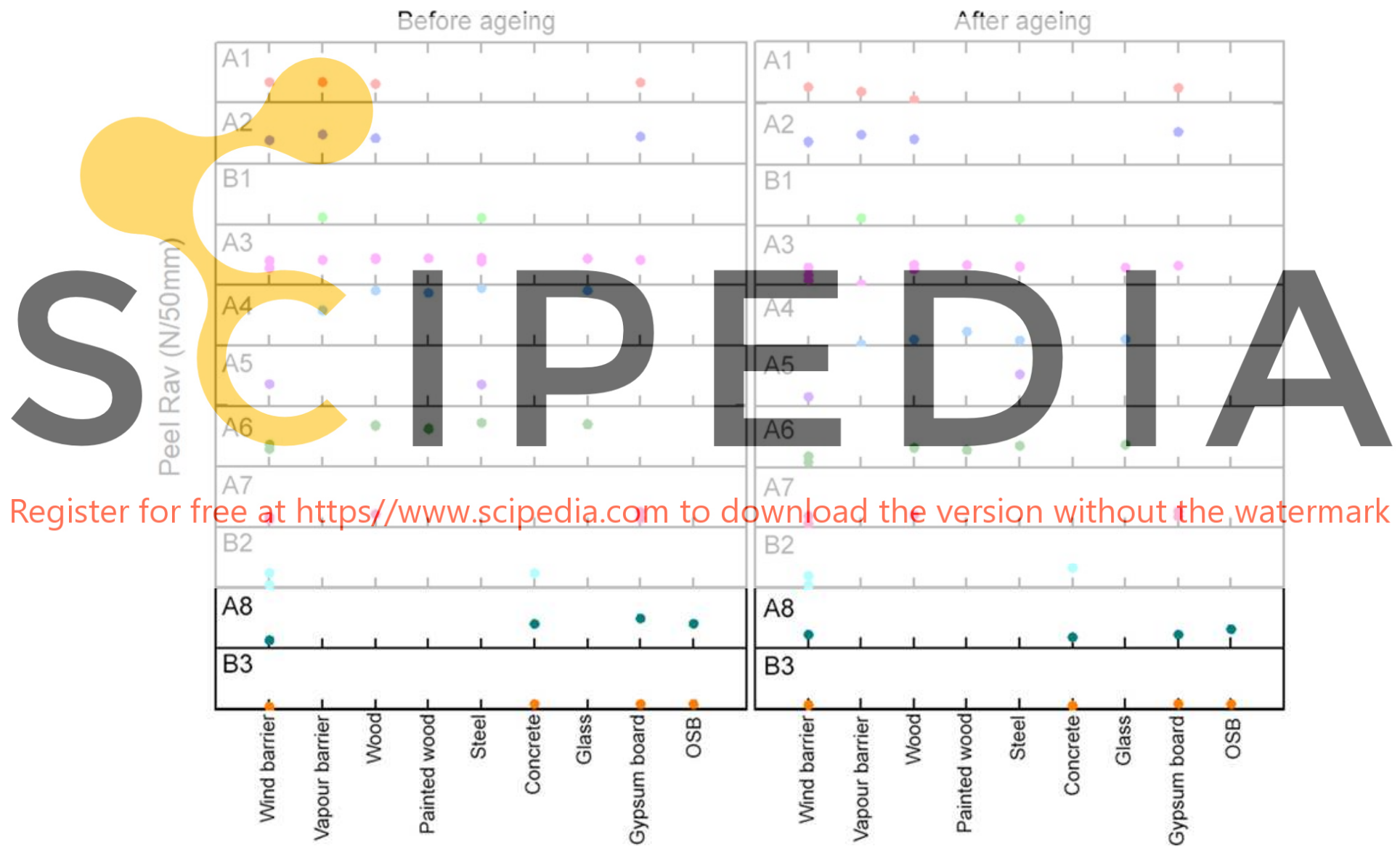

Figure 3. Stacked plot of peel resistance for 11 different adhesive tapes on various substrates. Each row contains data points from one tape product, labelled A1 - A8 for acrylic tapes and B1 - B3 for butyl rubber tapes. The yaxis for each row starts at $0 \mathrm{~N} / 50 \mathrm{~mm}$ and ends at $110 \mathrm{~N} / 50 \mathrm{~mm}$. 


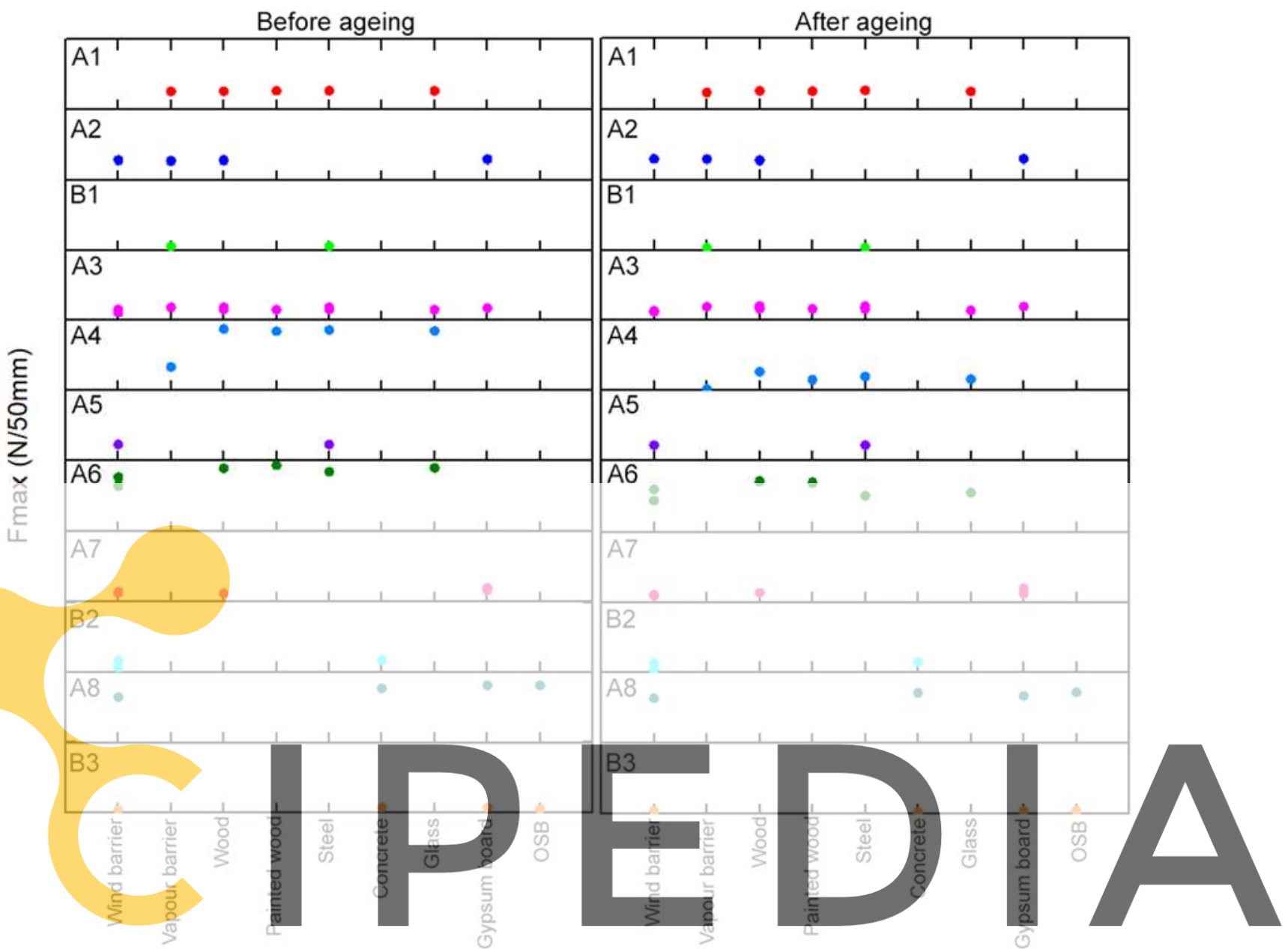

Figure 4. Stacked plot of shear resistance for 11 different adhesive tapes on various substrates. Each row

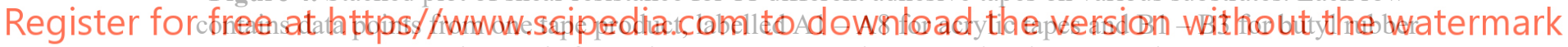
tapes. The y-axis for each row starts at $0 \mathrm{~N} / 50 \mathrm{~mm}$ and ends at $450 \mathrm{~N} / 50 \mathrm{~mm}$.
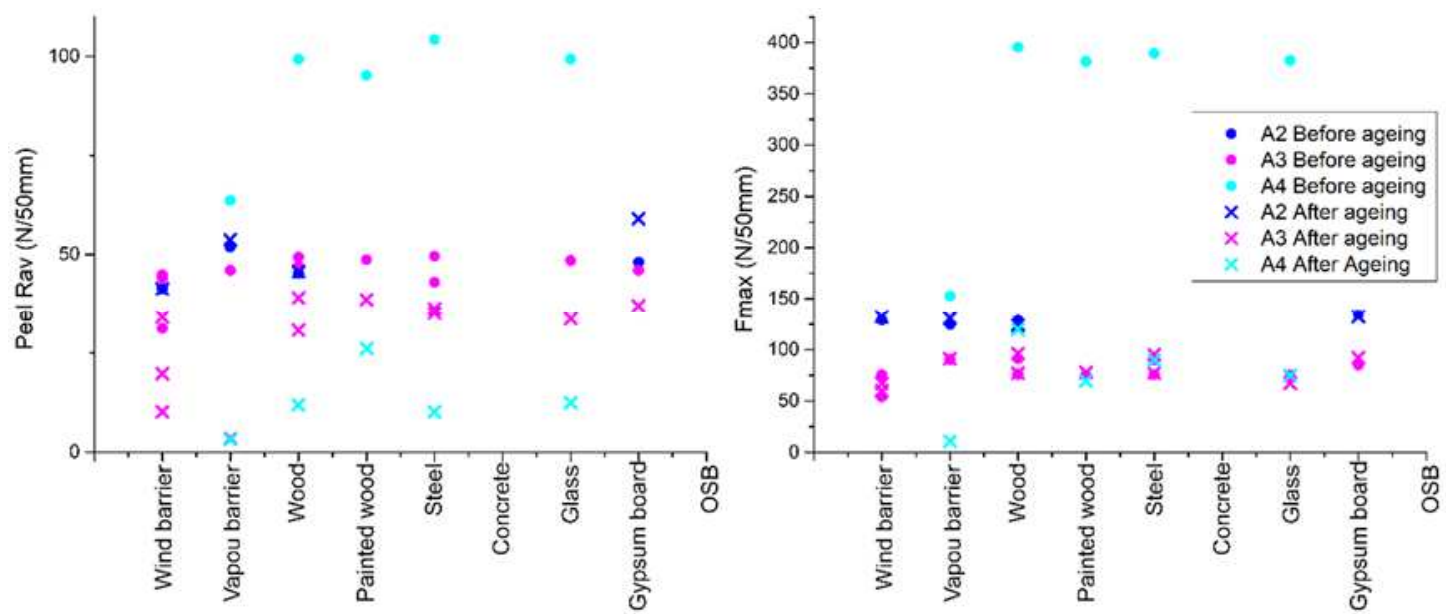

Figure 5. Peel and shear resistance before and after ageing for tapes A2, A3 and A4. 


\section{Conclusions}

- Peel resistance for non-aged samples was a poor predictor of peel resistance after ageing.

- There was a clear correlation between shear resistance for aged and non-aged samples.

- Peel resistance was a more sensitive evaluation parameter than shear strength to assess changes caused by artificial ageing.

- The measurement values for peel and shear resistance varied considerably more between different tapes than between different substrates. This was true for both nonaged and aged test specimens.

- More consideration should be made to include several different tapes in the experimental matrix when planning experiments to study the durability of adhesive tapes for building air-tightness systems.

\section{Acknowledgements}

This study was funded by the project 'TightEN - Durable adhesive airtight solutions for energy efficient building envelopes'. Research Council of Norway; Country: Norway; Grant number: 294894

\section{ORCII}

Malin Sletnes: https://orcid.org/0000-0001-8458-4653

Susanne Frank: https://orcid.org/0000-0002-4542-1402
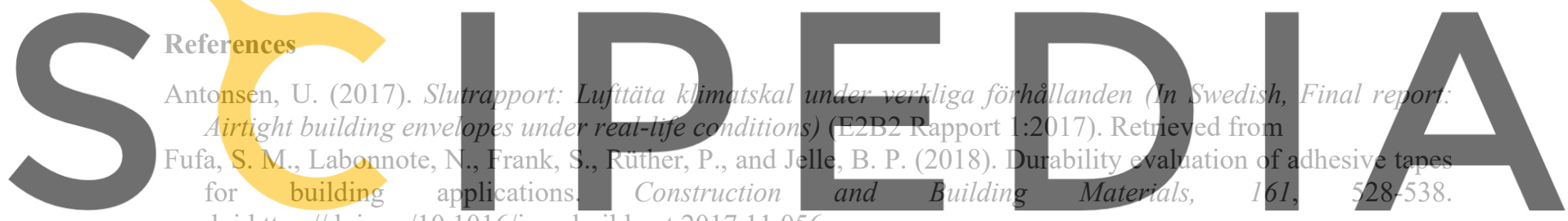

doi:https://doi.org/10.1016/i.conbuildmat.2017.11.056

Langmans, J., Desta, T. Z., Alderweireldt. L. and Roels, S. (2017). Durability of self-adhesive tapes for exterior

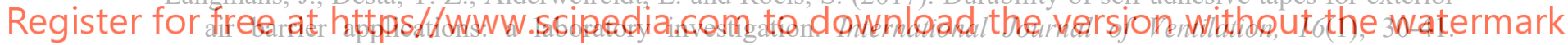
doi:10.1080/14733315.2016.1252154

Leprince, V., Moujalled, B. and Litvak, A. (2017). Durability of building airtightness, review and analysis of existing studies. Paper presented at the AIVC TightVent, Brussels.

Litvak, A., Allègre, F., Moujalled, B. and Leprince, V. (2019). Assessment of the durability of airtightness products in laboratory controlled conditions: development and presentation of the experimental protocol. Paper presented at the 40th AIVC Conference - From energy crisis to sustainable indoor climate - 40 years of AIVC, Ghent, Belgium. https://hal.archives-ouvertes.fr/hal-02335791 